\title{
Near-infrared light-responsive liposomal contrast agent for photoacoustic imaging and drug release applications
}

Kathyayini Sivasubramanian

Malathi Mathiyazhakan

Christian Wiraja

Paul Kumar Upputuri

Chenjie $\mathrm{Xu}$

Manojit Pramanik 


\title{
Near-infrared light-responsive liposomal contrast agent for photoacoustic imaging and drug release applications
}

\author{
Kathyayini Sivasubramanian, ${ }^{\mathrm{a}, \dagger}$ Malathi Mathiyazhakan, ${ }^{\mathrm{a}, \dagger}$ Christian Wiraja, ${ }^{\mathrm{a}}$ Paul Kumar Upputuri, ${ }^{\mathrm{a}}$ \\ Chenjie $\mathrm{Xu},{ }^{\mathrm{a}, \mathrm{b}, *}$ and Manojit Pramanik ${ }^{\mathrm{a}, *}$ \\ aNanyang Technological University, School of Chemical and Biomedical Engineering, 62 Nanyang Drive, Singapore \\ ${ }^{b}$ Nanyang Technological University, NTU-Northwestern Institute for Nanomedicine, 50 Nanyang Avenue, Singapore
}

\begin{abstract}
Photoacoustic imaging has become an emerging tool for theranostic applications. Not only does it help in in vivo, noninvasive imaging of biological structures at depths but it can also be used for drug release and therapeutic applications. We explore near-infrared light-sensitive liposomes coated with gold nanostars (AuNSs) for both imaging and drug release applications using a photoacoustic imaging system. Being amphiphilic, the liposomes lipid bilayer and the aqueous core enable encapsulation of both hydrophobic and hydrophilic drugs. The AuNSs on the surface of the liposomes act as photon absorbers due to their intrinsic surface plasmon resonance. Upon excitation by laser light at specific wavelength, AuNSs facilitate rapid release of the contents encapsulated in the liposomes due to local heating and pressure wave formation (photoacoustic wave). Herein, we describe the design and optimization of the AuNSs-coated liposomes and demonstrate the release of both hydrophobic and hydrophilic model drugs (paclitaxel and calcein, respectively) through laser excitation at nearinfrared wavelength. The use of AuNSs-coated liposomes as contrast agents for photoacoustic imaging is also explored with tissue phantom experiments. In comparison to blood, the AuNSs-coated liposomes have better contrast (approximately two times) at 2-cm imaging depth. @ 2016 Society of Photo-Optical Instrumentation Engineers (SPIE) [DOI: 10.1117/1.JBO.22.4.041007]
\end{abstract}

Keywords: photoacoustic imaging; drug release; liposomes; contrast agent; gold nanostars.

Paper 160561SSR received Aug. 18, 2016; accepted for publication Nov. 10, 2016; published online Dec. 1, 2016.

\section{Introduction}

Photoacoustic imaging (PAI) is a hybrid imaging modality that combines rich optical contrast and high ultrasonic resolution at depth. ${ }^{1,2}$ In PAI, short laser pulses are used to irradiate the sample. The absorption of light causes a small temperature rise and subsequently, due to thermoelastic expansion, pressure waves are generated in the form of acoustic waves [also known as photoacoustic (PA) waves]. By acquiring the PA waves using a wideband ultrasound detector at the tissue surface, images can be formed to reveal the internal structures and function of the tissue. PAI has a potential to image biological features from organelle to organs. ${ }^{1}$ The advantages of PAI include deeper penetration depth, good spatial resolution, and high soft tissue contrast. ${ }^{3}$ PAI provides anatomical, functional, metabolic, molecular, and genetic contrasts of vasculature, hemodynamics, oxygen metabolism, biomarkers, and gene expression. ${ }^{1,4}$ These unique advantages made PAI a potential tool for many applications in vascular biology, oncology, neurology, ophthalmology, dermatology, gastroenterology, cardiology, and so on. ${ }^{1,3}$

The PA image contrast is based on the optical absorption by endogenous molecules (hemoglobin, melanin, and so on); however, these molecules show relatively weaker light absorption in the near-infrared (NIR) region. Nevertheless, image contrast can be improved with the aid of exogenous contrast agents having strong optical absorption in the NIR wavelength region. Several

\footnotetext{
*Address all correspondence to: Manojit Pramanik, E-mail: manojit@ ntu.edu.sg Chenjie Xu, E-mail: cjxu@ntu.edu.sg

${ }^{\dagger}$ Authors contributed equally.
}

metallic, inorganic, organic nanoparticles, quantum dots, carbon nanotubes, and so on were demonstrated for deep-tissue PAI..$^{5-10}$ Contrast agents not only help to enhance the PA signals but are also useful for targeted molecular imaging, drug delivery, therapy, and so on. ${ }^{11-14}$ Research is in progress to find a stable, nontoxic, biocompatible, and yet powerful exogenous contrast agent for PAI. ${ }^{5,15}$

Among various nanocarriers, liposomes exhibit stable encapsulation of therapeutics and long circulation half-time, ${ }^{16,17}$ and these agents are clinically successful. ${ }^{18,19}$ The most conventional composition of liposomes contains dipalmitoylphosphatidyl choline (DPPC) as its main constituent. DPPC with a phase transition (transition from gel to liquid-crystalline state) temperature at around $41^{\circ} \mathrm{C}$ enables release of the encapsulated therapeutic from liposome in response to temperature. ${ }^{20}$ However, one of the major constraints of these liposomes is their inability to initiate the drug release at the target site due to inadequate temperature control in vivo. ${ }^{21}$ One of the various attempts to modulate the temperature in physiological conditions has resulted in stimuli responsive liposomes that can be externally manipulated by light. Simplicity, clinical adaptability, and unparalleled spatiotemporal control on the drug release being the key aspects, gold nanostructures are coupled with liposomes to make them better responsive to light. ${ }^{22,23}$ Earlier, a photoresponsive system encapsulated by gold nanoparticles (AuNPs) and the model drug calcein within temperature-sensitive liposomes by a simple thin film hydration method was developed.

$1083-3668 / 2016 / \$ 25.00$ (c) 2016 SPIE 
However, this method suffers from the low encapsulation of nanoparticles and the drug and is limited by penetration depth of visible light for clinical applications. ${ }^{24}$

In this work, we describe the synthesis and characterization of temperature-sensitive liposome coated with gold nanostars (AuNSs) with a strong absorption peak at $690 \mathrm{~nm}$. The strong absorption in the NIR window makes it suitable for PAI, making it an ideal dual functional imaging agent and drug delivery carrier for therapeutics. Coating the liposomes surface with AuNSs facilitates optimal encapsulation of the therapeutics within the liposomes, and the optical properties of AuNSs at NIR wavelength facilitate deep-tissue drug delivery. ${ }^{22,25}$ In addition, the proximity of the AuNSs to the lipid bilayer is more effective even in low intensities of light to cause the disruption of liposomes and subsequent drug release. Despite the advantages, till date, limited studies have been reported on gold-coated liposomes. Troutman et al. and Leung et al. reported the synthesis of gold-coated liposomes using ascorbic acid as the reducing agent. Although they were successful in demonstrating wavelength-selective drug release, the efficacy of the system was not tested on in vitro or in vivo systems. ${ }^{25}$ Gold-coated liposomes reported by Rengan et al. demonstrate light-triggered drug release by a photothermal effect, requiring a prolonged exposure of laser. ${ }^{26}$ Along with drug release applications, liposome-gold hybrid systems are being actively investigated for their applications in photothermal therapy and multimodal imaging. ${ }^{26,27}$ However, for PAI, gold-coated liposomes has not been reported. In this work, AuNS-coated liposomes as a potential exogenous contrast agent for deep-tissue PAT imaging is demonstrated. The near instantaneous controlled drug delivery from AuNSs-coated liposomes using PA effect is also explored.

\section{Materials and Methods}

1,2-Dipalmitoyl-sn-glycero-3-phosphocholine (DPPC), 1-palmitoyl-2-hydroxy-sn-glycero-3 phosphocholine (MPPC), and 1, 2distearoyl-sn-glycero-3-phosphoethanol-amine- $N$-[carboxy (polyethylene glycol)-2000] (ammonium salt) (DSPE-PEG2000) were purchased from Avanti Polar Lipids (Alabama). $\mathrm{HAuCl}_{4}$ and Paclitaxel were purchased from ACROS Organics. Fresh mouse blood was collected from the animal facility center, Nanyang Technological University. All other chemicals unless otherwise mentioned were purchased from Sigma-Aldrich. Deionized (DI) water was purified by a Millipore Milli-DI water purification system.

\subsection{Synthesis of Liposomes Coated with Gold Nanostars}

Temperature-sensitive liposomes were first prepared by the conventional thin film hydration method as reported previously. ${ }^{28}$ Briefly $7.95,0.65$, and $1.39 \mathrm{mg} / \mathrm{mL}$ of DPPC, MPPC, and DSPE-PEG2000, respectively, were dissolved in chloroform (in a molar ratio of 86:10:4). The organic phase was evaporated at $37^{\circ} \mathrm{C}$ under reduced pressure in a rotary evaporator (Buchi, Switzerland) to form the lipid bilayer. For paclitaxel-loaded liposomes (PTX-L), $1 \mathrm{mg}$ of PTX was dissolved in the organic phase. For calcein-loaded liposomes (Cal-L), the thin lipid film was hydrated with 1-mL calcein solution $(60 \mathrm{mM}, 7.4 \mathrm{pH})$. The plain liposomes (PL) were hydrated with PBS. The liposomes were then subjected to size extrusion using 200-nm polycarbonate membrane filters (Avanti Polar Lipids). The unencapsulated calcein solution was removed by gel filtration chromatography with PD-10 desalting columns (GE Healthcare). The liposomes were then centrifuged at $12,000 \mathrm{rpm}$ at $4^{\circ} \mathrm{C}$ for $60 \mathrm{~min}$ to form a concentrated pellet.

To coat the liposomes with AuNSs, a liposomal pellet with a total lipid concentration of $2 \mathrm{mg}$ was reconstituted in $1 \mathrm{~mL}$ of 1 M HEPES buffer (pH 7.4). An amount of $50 \mu \mathrm{L}$ of $\mathrm{HAuCl}_{4}$ was added to the solution and was left at room temperature in dark under constant shaking at $400 \mathrm{rpm}$. After $1 \mathrm{~h}$, the liposomal solutions coated with AuNSs were centrifuged at 12,000 rpm $4^{\circ} \mathrm{C}$ for $30 \mathrm{~min}$ to remove the free AuNSs and excess HEPES buffer. PLs coated with AuNSs (AuL), calcein-loaded liposomes coated with AuNSs (Cal-AuL), and PTX-loaded liposomes coated with AuNSs (PTX-AuL) were thus formed by the above procedure. The liposomes were stored at $4^{\circ} \mathrm{C}$ and were used within $48 \mathrm{~h}$.

\subsection{Characterization of Liposomes Loaded with AuNSs}

The hydrodynamic diameter, polydispersity, and zeta potential of liposomes were measured using a dynamic light scattering (DLS) technique (ZetaPALS analyzer, BIC). An average of six measurements was reported for each sample. The concentration of gold in liposomes was quantified by inductively coupled plasma spectrometer (ICP, Prodigy ICP spectrometer). An amount of $100 \mu \mathrm{L}$ of liposome was digested overnight in a mixture of $\mathrm{HCl}$ and $\mathrm{HNO}_{3}$ (in a ratio 3:1). The sample was then diluted to $10 \mathrm{~mL}$ and was quantified against the gold standards. The morphology of the liposomes and the presence of AuNSs coated on the lipid bilayer surface were determined by transmission electron microscopy (TEM). A $10 \mu \mathrm{L}$ of sample solution was placed on 200 mesh formvar carbon-coated copper grids (FCF-200-Cu, Electron Microscopy Sciences) and was airdried. After negative staining with $3 \%$ uranyl acetate for $1.5 \mathrm{~min}$, the grids were washed with DI water. Finally, the grid samples were air-dried overnight and were imaged using FEI Tecnai G2 Spirit Bio-TWIN electron microscope, operated at $120 \mathrm{kV}$. The morphology of the AuNSs was confirmed using high-resolution TEM imaging. For this, the liposomes were lysed with Triton X-100 and AuNSs were collected after centrifuging the samples at $15,000 \mathrm{~g}$ for $15 \mathrm{~min}$ at $4^{\circ} \mathrm{C}$. The AuNSs were then redissolved in DI water and $5 \mu \mathrm{L}$ of the AuNSs solution was placed on 200 mesh formvar carbon-coated copper grids (FCF-200-Cu, Electron Microscopy Sciences). The grids were completely dried before imaging with TEM (FEI G2 spirit, $120 \mathrm{kV}$, America). Phospholipid assay was performed to determine the total lipid concentration by mass. Loading efficiency was estimated from the standard curves constructed for known concentration of phospholipids, calcein, and paclitaxel, at an excitation max of 535, 480, and $230 \mathrm{~nm}$, respectively. The loading efficiency was determined by

$$
\mathrm{LE}=\frac{\text { Wt of calcein encapsulated }}{\text { Wt of final total lipids }} \times 100 \text {. }
$$

\subsection{Cell Culture}

B16-F10 mouse melanoma cells (ATCC) were cultured and maintained in Dulbecco's modified eagle medium (DMEM, high glucose $4.5 \mathrm{~g} / \mathrm{L}$, and $4.0 \mathrm{mM}$ L-glutamine) supplemented with $10 \%$ FBS and penicillin/streptomycin $(100 \mathrm{U} / \mathrm{ml})$ at $37^{\circ} \mathrm{C}$ with $5 \% \mathrm{CO}_{2}$. 


\subsection{Photoacoustic Spectrum of Liposomes Loaded with AuNSs}

An optical parametric oscillator, OPO (Continuum, Surelite), pumped by a Q-switched 532-nm Nd:YAG laser, was used as an excitation source for PA spectrum measurements. It generates 5-ns duration pulses at $10-\mathrm{Hz}$ repetition rate with wavelengths tunable from 670 to $2500 \mathrm{~nm}$. The solution containing liposomes coated with AuNSs (AuL) was placed inside a lowdensity polyethylene (LDPE) tube with an inner diameter of $0.59 \mathrm{~mm}$ and an outer diameter of $0.78 \mathrm{~mm}$. The sample containing tube and the single-element ultrasound transducer (UST) (V323-SU/2.25 MHz, 13-mm active area, and 70\% nominal bandwidth, Panametrics, Olympus) were immersed in water for coupling of PA signals to UST. The tube was irradiated with wavelengths ranging from 670 to $900 \mathrm{~nm}$ with a 10-nm step size. Respective PA signals were collected using the UST, and these signals were subsequently amplified and band pass filtered by an amplifier/filter unit (A/F) (Olympus-NDT, 5072PR). Finally, the output from the A/F unit was digitized with a data acquisition card (GaGe, compuscope 4227) and stored in the computer. Peak-to-peak PA signal was then normalized with the laser energy for each wavelength and was plotted against the wavelength to generate the PA spectrum.

\subsection{Photoacoustic Signal from Liposome and Animal Blood}

The PA spectrum of liposomes coated with AuNSs (AuL) shows peak at $690 \mathrm{~nm}$. So, for further PA experiments, the laser was tuned to $690 \mathrm{~nm}$ and the incident laser energy density was maintained at $5 \mathrm{~mJ} / \mathrm{cm}^{2}$. To measure PA signal, the AuNSs-coated liposome sample/animal blood was placed inside a $0.59-\mathrm{mm}$ diameter LDPE tube. The sample containing tube was irradiated with a 690-nm laser beam. Respective PA signals from liposome and blood samples were collected using the $2.25-\mathrm{MHz}$ UST.

\subsection{Deep-Tissue Photoacoustic Imaging Experiment}

The LDPE tubes $(\sim 1 \mathrm{~cm}$ long) filled with fresh mouse blood and AuNSs-coated liposomes $(2 \mathrm{mg} / \mathrm{mL})$ were embedded in a chicken breast tissue. A photoacoustic tomography (PAT) imaging system was used for tissue imaging experiments. ${ }^{29}$ The tissue surface was irradiated with an expanded 690-nm laser beam having energy density $5 \mathrm{~mJ} / \mathrm{cm}^{2}$ (<ANSI safety limit $20 \mathrm{~mJ} / \mathrm{cm}^{2}$ ), and the PA signals were collected by continuously rotating the $2.25-\mathrm{MHz}$ UST around the sample in a circular geometry. The scan time was $1 \mathrm{~min}$. PA signals were collected when the tubes were placed at a depth of 1 and $2 \mathrm{~cm}$ beneath the laser-illuminated chicken tissue surface. The cross-sectional PAT images were reconstructed using a delay-and-sum algorithm.

\subsection{Photoacoustic Imaging of Melanoma Cells}

PAI of the B16F10 melanoma cells was done using a clinical ultrasound-PAI system. The cells were labeled with AuNSscoated liposomes with a total lipid concentration of $100 \mu \mathrm{g} /$ $\mathrm{mL}$ for $48 \mathrm{~h}$, and unlabeled cells were used as control. The labeled and the unlabeled cells were then trypsinized and centrifuged at $400 \mathrm{~g}$ for $5 \mathrm{~min}$ to obtain the cell pellet. The experimental setup uses a clinical research ultrasound system (ECUBE 12R, Alpinion, South Korea) with PAI capability. In order to operate the system in the PA mode, laser excitation needs to be provided as well as a trigger, which synchronizes the excitation of the laser and the signal acquisition by the UST. ${ }^{30}$ With a final concentration of $1 \times 10^{5}$ cells $/ \mathrm{mL}$ in $1-\mathrm{mL}$ transparent tubes at an imaging depth of $2 \mathrm{~cm}$, the cells were irradiated at $690 \mathrm{~nm}$. Real-time images were acquired at a frame rate of 5 frames per second (fps). The PA signals were acquired using a linear array transducer (L3-12 transducer), consisting of 128 array elements with a center frequency of $\sim 8.5 \mathrm{MHz}$.

\subsection{Drug Release Studies}

A 1-mL calcein-encapsulated AuNSs-coated liposome solution (Cal-AuL) was taken in a quartz cuvette and was irradiated for varying time points ( 0 to $60 \mathrm{~s}$ ) with the OPO laser with an energy density of $10 \mathrm{~mJ} / \mathrm{cm}^{2}$. A $10-\mu \mathrm{L}$ liposomal solution was collected after every $5 \mathrm{~s}$ and was replaced with fresh aliquots. Collected samples were transferred to an Eppendorf tube containing distilled water and were placed onto an ice box to avoid further release. The samples were centrifuged at 15,000 rpm to remove AuNSs-coated liposomes and the supernatant was transferred to 96 well plates. The percentage of calcein released was quantified by measuring the fluorescence at an excitation and emission wavelengths of 480 and $520 \mathrm{~nm}$, respectively, using a fluorescence spectrophotometer (H4 Synergy). The experiment was repeated with calcein-encapsulated liposome without the AuNSs (Cal-L). Both Cal-L and Cal-AuL were treated at $37^{\circ} \mathrm{C}$, and the amount of calcein released was measured as a control. In the same way, PTX release assay was also performed using PTX-encapsulated AuNSs-coated liposome solution (PTXAuL) and PTX-encapsulated liposome without the AuNSs (PTXL). The percentage of PTX released was quantified by measuring the absorbance at $230 \mathrm{~nm}$ using an UV spectrophotometer (Spectramax). Both PTX-L and PTX-AuL were treated at $37^{\circ} \mathrm{C}$, and the amount of PTX released was measured as a control.

\subsection{Qualitative Analysis of In Vitro Calcein Release}

For in vitro experiments, B16F10 mouse melanoma cells were incubated with Cal-AuL (with a final concentration of $40-\mu \mathrm{g}$ total lipids $/ \mathrm{mL}$ ) and were incubated for $6 \mathrm{~h}$ at $37^{\circ} \mathrm{C}$ with $5 \%$ $\mathrm{CO}_{2}$. The cells were washed twice with PBS and then irradiated with the OPO laser at $690 \mathrm{~nm}$ for $30 \mathrm{~s}$ per group with an energy density of $10 \mathrm{~mJ} / \mathrm{cm}^{2}$. PLs, free calcein, and Cal-L-treated groups were used as controls. To verify the calcein release from the liposomes, the cells were imaged using a LX71 inverted fluorescence microscope (Olympus) and a Retiga-2000R CCD camera, 1-h postlaser treatment. It is to be noted that the settings (500-ms exposure time and $3 \times$ gain) for the all green fluorescent images, at $20 \times$ image magnification was kept constant throughout the imaging. Finally, the images were processed using the ImageJ software to normalize background signal. The calcein release intensity was quantified using the fluorescence microplate reader with an excitation and emission wavelengths of 480 and $515 \mathrm{~nm}$, respectively.

\subsection{Cytotoxicity Assay}

The B16F10 mouse melanoma cells were incubated with paclitaxel-loaded liposomes coated with AuNSs (with a final concentration of 40- $\mu \mathrm{g}$ total lipids $/ \mathrm{mL}$ ) and were incubated for $6 \mathrm{~h}$ at $37^{\circ} \mathrm{C}$ with $5 \% \mathrm{CO}_{2}$. The cells were washed thrice with PBS and then irradiated with an OPO laser at $690 \mathrm{~nm}$ for $30 \mathrm{~s}$ per group with an energy density of $15 \mathrm{~mJ} / \mathrm{cm}^{2}$. PLs, free PTX, and PTXL-treated groups were used as controls. To test the effectiveness 
of the release of paclitaxel, cell viability assay was performed using Alamar Blue, $24 \mathrm{~h}$ postlaser treatment.

\section{Results and Discussion}

Temperature-sensitive liposomes were prepared using the conventional thin film hydration method and coated with AuNSs by reducing the gold precursor, $\mathrm{HAuCl}_{4}$ using HEPES buffer. HEPES was found to reduce the $\mathrm{Au}^{3+}$ ions in $\mathrm{HAuCl}_{4}$ to $\mathrm{Au}^{0}$, thus forming AuNSs. ${ }^{31}$ This zerovalent state leads to the deposition of AuNSs on the surface of the liposomes. ${ }^{32}$ The liposome was characterized as shown in Fig. 1. The presence of AuNSs on the surface of the liposomes imparts characteristic optical properties to the AuNSs-coated liposomes making it responsive to light. The maximum absorbance of light occurred at $690 \mathrm{~nm}$ measured from the UV-vis spectroscopy as shown in Fig. 1(a). The PLs and AuNSs-coated liposomes were characterized for their size, surface potential, and morphology. The hydrodynamic diameter measured by the DLS method was found to be $141.4 \pm 0.5$ and $113.2 \pm 0.7 \mathrm{~nm}$ as shown in Fig. 1(b), while the surface charge was $-28.38 \pm 1.25$ and $-22.37 \pm 0.23 \mathrm{mV}$ for PLs and AuNSs-coated liposomes, respectively. The TEM images indicated the presence of AuNSs on the surface of the liposomes and the size of the AuNSs liposomes from the TEM images was found to be $96.77 \mathrm{~nm}$ [Fig. 1(c)], which is comparable to the hydrodynamic diameter measured. The HR-TEM images clearly revealed the branched star-shaped morphology of the AuNSs as shown in Fig. 1(d). From the ICP results, the concentration of gold in AuNSs-coated liposomes was found to be $160 \mu \mathrm{M}$. The loading efficiencies of calcein and PTX were found to be $24 \%$ and $25 \%$, respectively.
The PA spectrum revealed that there is a strong PA signal at $690 \mathrm{~nm}$ as shown in Fig. 2(a), indicating high optical absorbance at this wavelength. Based on this observation, the laser excitation was fixed at $690 \mathrm{~nm}$ for further experiments. At $690 \mathrm{~nm}$, it was found that the PA signal from $2 \mathrm{mg} / \mathrm{mL}$ AuNSs-coated liposomes was two times stronger than that from the animal blood as shown in Fig. 2(b). The peak-to-peak PA signal from blood and AuNSs-coated liposomes were found to be 0.21 and $0.45 \mathrm{~V}$, respectively. The potential use of AuNSs-coated liposomes as PA contrast agent and their effective imaging depth at a wavelength of $690 \mathrm{~nm}$ were tested. As shown in Fig. 2(d), two LDPE tube phantoms were filled with fresh mouse blood and AuNSscoated liposomes $(2 \mathrm{mg} / \mathrm{mL})$, respectively. They were sandwiched between chicken breast tissue of varying depths (1 and $2 \mathrm{~cm}$ ). Figures 2(e) and 2(f) show the cross-sectional PAT images of the tubes. The signal-to-noise ratio (SNR) values of blood and AuNSs-coated liposomes at $1 \mathrm{~cm}$ depth was 13 and 25, respectively, as shown in Fig. 2(e). Similarly, SNR values of blood and AuNSs-coated liposomes at $2 \mathrm{~cm}$ depth were 6 and 14, respectively, as shown in Fig. 2(f). Our results indicate that AuNSs-coated liposomes are promising contrast agents for PAT, providing good PA signal enhancement (approximately two times) and image contrast in biological tissues even at $2-\mathrm{cm}$ depth.

The tube containing the B16F10 melanoma cells in the media is shown in Fig. 3(a). The combined ultrasound and B-scan PA image (beam-formed image) of the tubes containing the unlabeled and the labeled melanoma cells are shown in Figs. 3(b) and 3(c), respectively. The grayscale image represents the ultrasound image and the colors represent the PA image. Melanin has
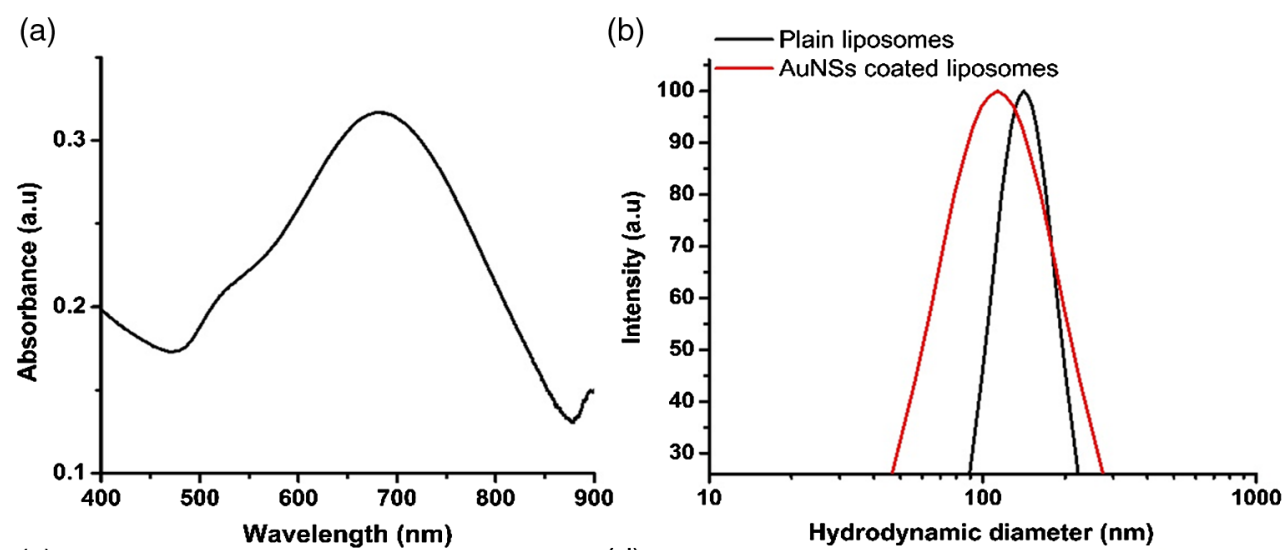

(c)

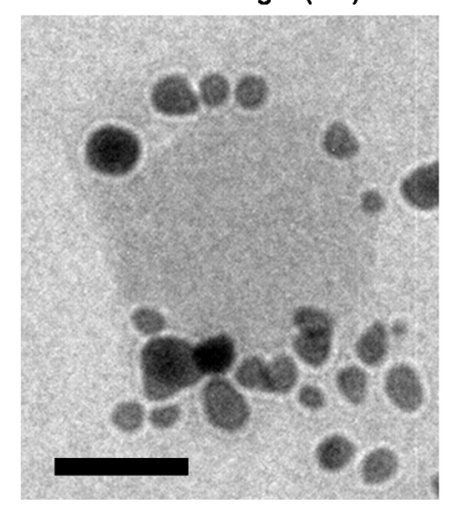

(d)

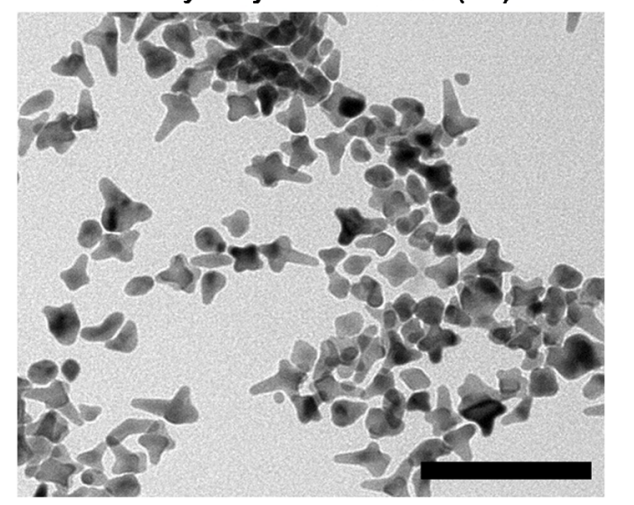

Fig. 1 Characterization profile of AuNSs-coated liposomes represented by the (a) UV-vis absorption spectrum of AuNSs-coated liposomes, (b) hydrodynamic diameter of AuNSs-coated liposomes and PLs, (c) TEM image of AuNSs-coated liposomes, and (d) HR-TEM image of AuNSs. Scale bar: $50 \mathrm{~nm}$. 

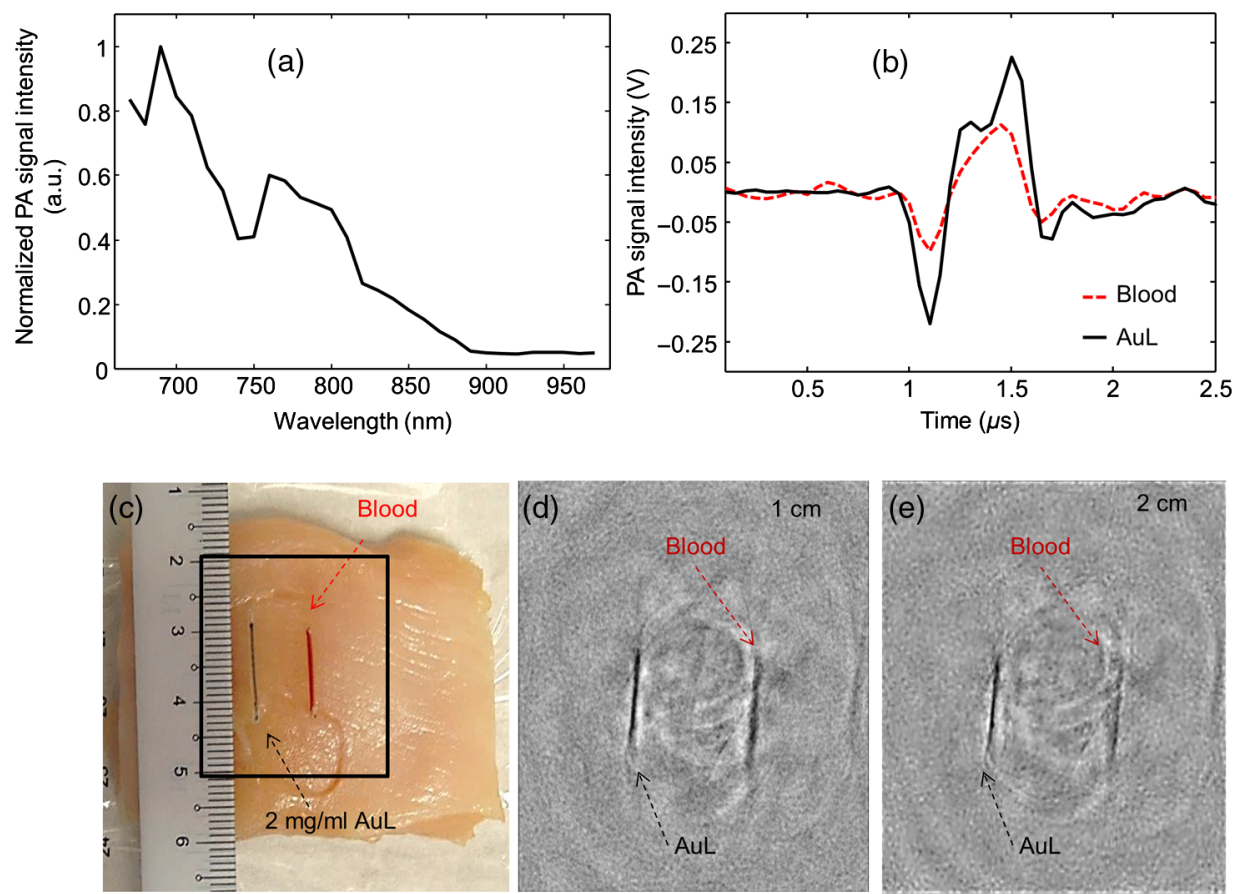

Fig. 2 PA spectrum and imaging: (a) PA spectrum of AuNSs-coated liposomes (AuL) in the NIR wavelength range, (b) PA signals collected from blood and AuNSs-coated liposomes, (c) photograph of the LDPE tubes filled with $2 \mathrm{mg} / \mathrm{mL}$ AuNSs-coated liposomes and animal blood placed inside (sandwiched) chicken breast tissue (the top layer was removed to take the picture), (d, e) cross-sectional PAT images of the tubes at (d) $1 \mathrm{~cm}$ and (e) $2 \mathrm{~cm}$ imaging depth.

an intrinsic PA signal. It has a good contrast against blood in a wide range of wavelengths ( 600 to $800 \mathrm{~nm}$ ). The bright red spots on the image show the melanoma cells. It can be seen that in both the labeled and the unlabeled samples, the cells can be imaged. The difference in the signal intensity might not be so obvious from the PA/US image because the system normalizes the images by default. If the PA images alone are extracted from it, PA signal would be more pronounced and obvious. This can be noted from Figs. 3(d) and 3(e), which show the normalized
PA images of both the labeled and the unlabeled melanoma cells. Also, for clarity the SNR from both the images is reported in Fig. 3(f). The SNR was defined as the amplitude of the PA signal from the melanoma cells divided by the standard deviation of the background noise, $\mathrm{SNR}=V / n$, where $V$ is the PA signal amplitude and $n$ is the standard deviation of the background noise. As shown in Fig. 3(f), the SNR of the unlabeled B16F10 mouse melanoma cells is 45 , while that of the AuNSscoated liposomes labeled B16F10 mouse melanoma cells is 174 .

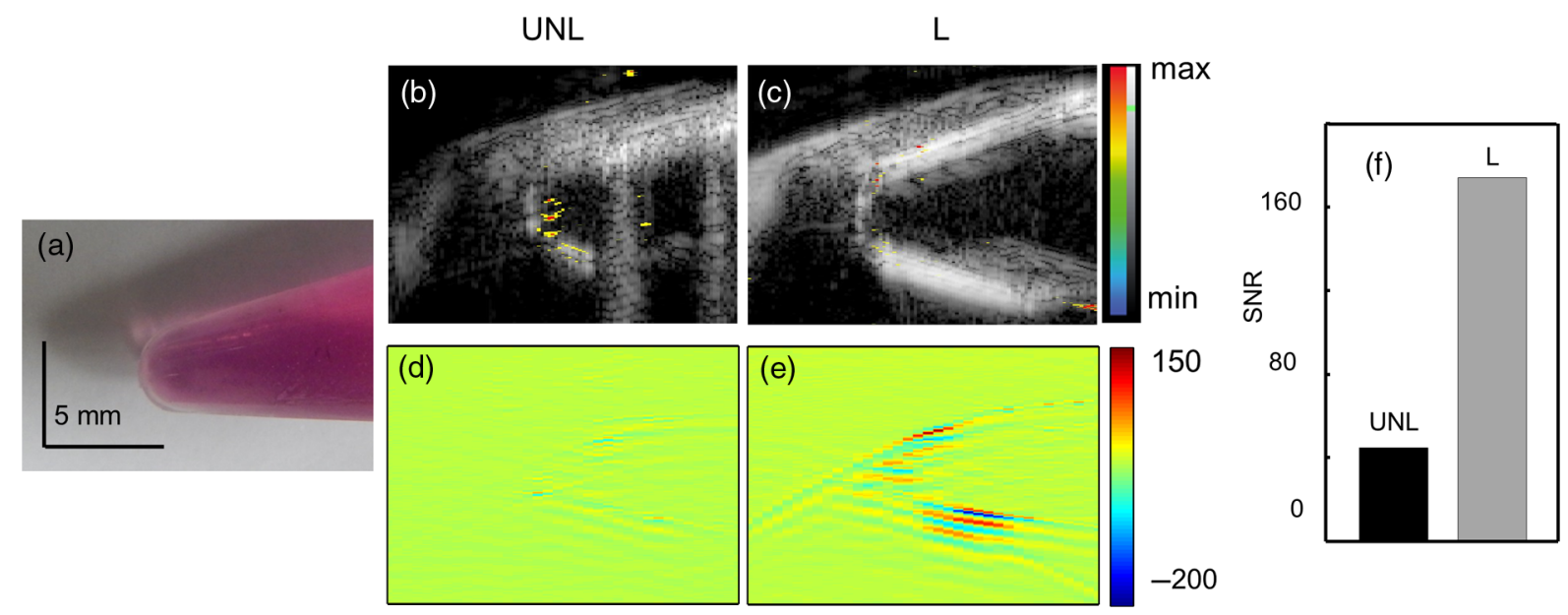

Fig. 3 (a) Photograph of a tube containing B16F10 mouse melanoma cells in media, (b) combined ultrasound and B-scan PA image unlabeled B16F10 mouse melanoma cells, (c) combined ultrasound and Bscan PA image of AuNSs-coated liposomes labeled B16F10 mouse melanoma cells, (d) B-scan PA image of unlabeled B16F10 mouse melanoma cells, (e) B-scan PA image of AuNSs-coated liposomes labeled B16F10 mouse melanoma cells, and (f) graph comparing the SNR values of unlabeled B16F10 mouse melanoma cells (UNL) and AuNSs-coated liposomes labeled B16F10 mouse melanoma cells (L). 
This 3.8 time better signal intensity of the labeled cells further confirms that AuNSs-coated liposomes can be used as a PA contrast agent for cell labeling and tracking.

Next, the drug release capability of the liposomes was tested. Cal-AuL and PTX-AuL solutions were exposed to the NIR pulsed laser for varying time points ( 0 to $60 \mathrm{~s}$ ) with an energy density of $10 \mathrm{~mJ} / \mathrm{cm}^{2}$. The percentage of drug release from liposomes and AuNS-coated liposomes is shown in Figs. 4(a) and 4(b), respectively. From the figures, it can be observed that the presence of AuNS enabled 72.09 $\pm 0.88 \%$ and $76.08 \pm 3.28 \%$ release of encapsulated calcein and PTX, respectively, within $60 \mathrm{~s}$ under the laser exposure. While the liposomes without AuNSs had only $\sim 10 \%$ drug release under the same experimental conditions.

Fluorescence imaging using B16F10 melanoma cells was done to demonstrate the release of calcein from the liposomes triggered by light-induced pressure waves (PA waves). B16F10 melanoma cells were incubated with free calcein solution $(120 \mu \mathrm{M})$, liposomes containing calcein, and AuNSs-coated liposomes containing calcein overnight. After washing the cells with PBS to remove the particles that were not taken up by the cells, each of the treated groups was exposed to a laser at $690 \mathrm{~nm}$ at $10 \mathrm{~mJ} / \mathrm{cm}^{2}$ for $30 \mathrm{~s}$. When subject to a laser source at $690 \mathrm{~nm}$, the absorbed light causes a temperature rise, which generates pressure waves (PA waves). These pressure waves lead to the disruption of liposomes, releasing the encapsulated drug. ${ }^{33}$ Laser-untreated groups were compared against the laser-treated groups. This is shown in Fig. 5. It was observed that free calcein stained the cells in both laser-treated and -untreated groups; no fluorescence was observed in both groups in the case of cells incubated with liposomes containing calcein. However, in the cells incubated with AuNSs-coated liposomes containing calcein, a significant increase in fluorescence was observed in laser-treated groups while no fluorescence was observed in groups unexposed to laser. As reported earlier, the local temperature increase in the nanoparticle microenvironment generates microbubble cavitation that disrupts the liposomal membrane to release the encapsulated drug. ${ }^{22,24}$ Likewise, the presence of AuNSs in the liposomes results in calcein release due to microbubble cavitation. It is also to be noted that the rise in temperature of the bulk solution containing the same concentration of AuNSs-coated liposomes was found to be less than $4^{\circ} \mathrm{C}$ upon laser exposure of about $10 \mathrm{~min}$. This further validates that the encapsulated drug is released by the liposome disruption due to the pressure wave generation and eliminates the possibility of drug release due to a rise in temperature of the bulk liposomal solution. At a concentration of $60 \mathrm{mM}$, calcein selfquenches within the liposomes and the cells are nonfluorescent. As the laser triggers the AuNSs-coated liposomes containing calcein, the released calcein stains the cells and can be detected as their fluorescence recovers.

To test the biocompatibility of the AuNSs-coated liposomes, B16F10 mouse melanoma cells were incubated with AuNSs, free drug paclitaxel, liposomes loaded with paclitaxel, AuNSscoated liposomes loaded with paclitaxel for $24 \mathrm{~h}$. The cells were then washed with PBS, and the cell viability was assessed using Alamar blue assay against the untreated cells. Similarly, cells treated with laser at $690 \mathrm{~nm}$ for $30 \mathrm{~s}$ were tested for viability $24 \mathrm{~h}$ post laser treatment to see the efficacy of the drug release. These results are shown in Fig. 6. It was found that the cells that were not exposed to laser did not show any significant changes in the viability. While the cells treated with PTX showed significant decreases in cell viability in both control and laser-treated groups, about $20 \%$ cell death was observed from cells treated with AuNSs-coated liposomes without PTX. This clearly indicates that the microbubble cavitation generated due to the presence of AuNSs in the liposomes has led to the release of PTX into the cell cytoplasm, which in turn resulted in cell death. ${ }^{34}$ However, the highest percentage of cell death was observed from cells treated with AuNSs-coated liposomes containing paclitaxel upon laser exposure. Cells treated with liposomes with paclitaxel did not show significant changes in the cell viability ensure stable encapsulation of PTX within liposomes. This shows that the AuNSs cause significant drug release from the liposomes.

PAI can be used for theranostic applications when used with a suitable contrast agent. Here, we explore the theranostic potential of NIR light-sensitive liposomes coated with AuNSs for both imaging and drug release applications using PAI system. The liposome being amphiphilic can encapsulate both hydrophobic and hydrophilic molecules. Irradiation by laser at a particular wavelength triggers the rapid release of the contents encapsulated in the liposomes due to local heating and pressure wave formation (PA wave) by the AuNSs. The pressure waves
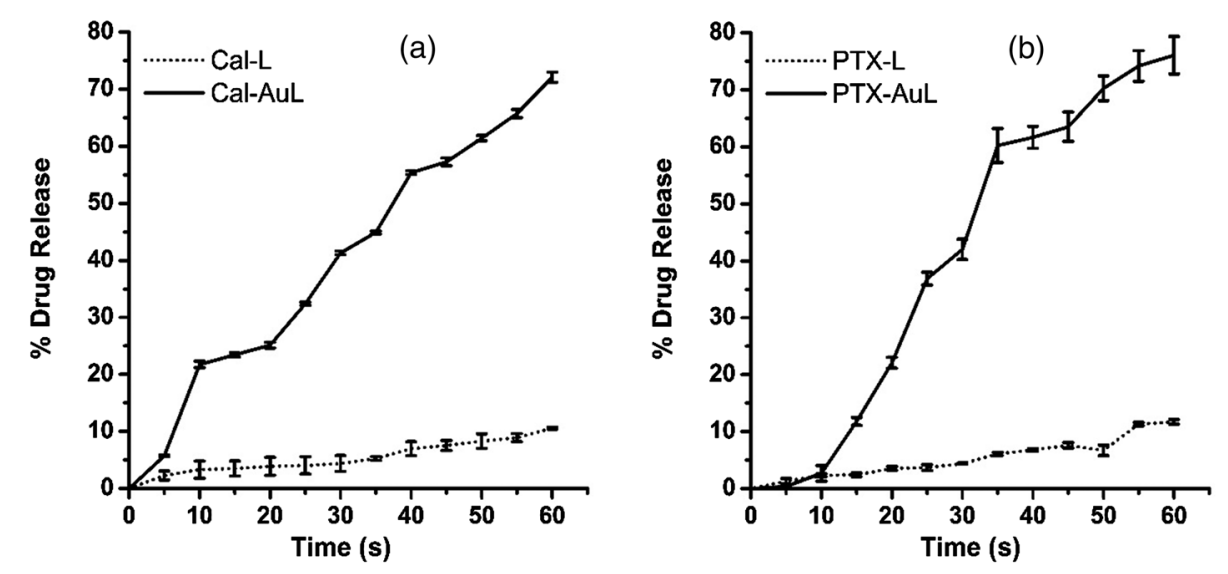

Fig. 4 Percentage of drug release (a) release of calcein from Cal-AuL and Cal-L. (b) Release of PTX from PTX-AuL and PTX-L upon laser irradiation at $690 \mathrm{~nm}$ with an energy density of $10 \mathrm{~mJ} / \mathrm{cm}^{2}$ at a pulse repetition rate of $10 \mathrm{~Hz}$. 


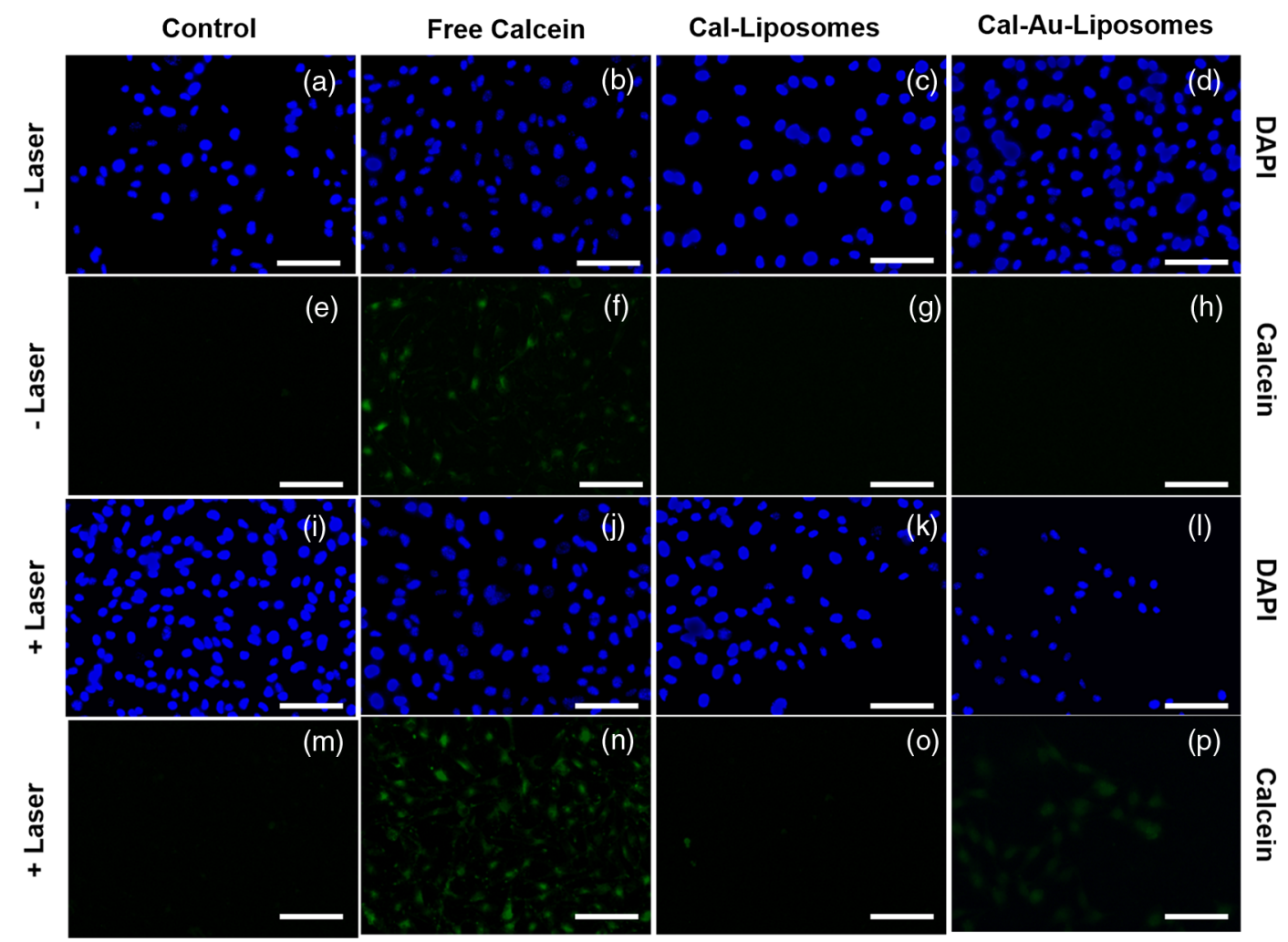

Fig. 5 In vitro release of calcein from Cal-AuL into the cytoplasm of B16F10 melanoma cells with and without laser exposure. The cells were irradiated with a laser for $30 \mathrm{~s}$ at an energy density of $10 \mathrm{~mJ} / \mathrm{cm}^{2}$. (a-d) The DAPI-stained cell nuclei of control group without any laser exposure. (e-h) The calcein-stained cell cytoplasm of control group without any laser exposure. (i-l) The DAPI-stained cell nuclei of lasertreated group. $(m-p)$ The calcein-stained cell cytoplasm of laser-treated group. $(p)$ The cells stained with calcein released from Cal-AuL after laser exposure. Blue, nuclei; green, cell cytoplasm. Scale bar: $100 \mu \mathrm{m}$.

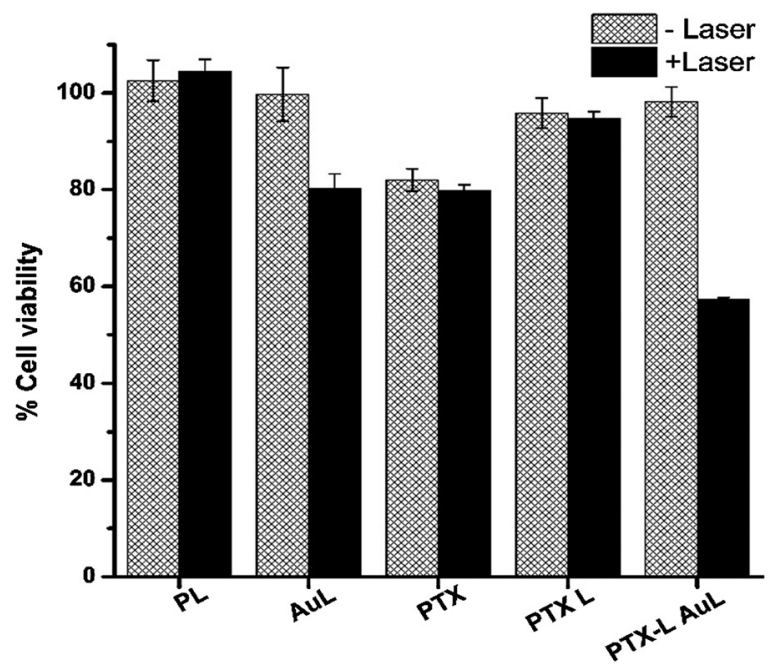

Fig. 6 Cell viability study of B16F10 melanoma cells treated with PTX Au-liposomes with and without laser exposure. The sample was irradiated with laser for $30 \mathrm{~s}$ at $15 \mathrm{~mJ} / \mathrm{cm}^{2}$ energy density. (PL, plain liposomes, AuL, AuNS-coated liposomes, PTX, free drug paclitaxel, PTX L, liposomes loaded with paclitaxel, PTX-L AuL, AuNS-coated liposomes loaded with paclitaxel). can also be used for PA imaging as well. However, further in vivo studies on small animals need to be done to prove the efficacy of this system for clinical applications.

\section{Conclusion}

In this work, we have demonstrated that AuNSs-coated liposomes show strong absorption in the NIR region and can be used as contrast agent for PAI of deep tissues. The drug release study promises that it can be used to deliver both hydrophilic and hydrophobic drugs effectively. Thus we present AuNSscoated liposomes as a dual modal therapeutic and contrast agent responsive to NIR light. Further use of these particles for in vivo small animal models is under investigation for its potential use in the clinics.

\section{Disclosures}

No conflicts of interest, financial or otherwise, are declared by the authors.

\section{Acknowledgments}

We thank Dr. Yang Cheng (Tsinghua University, China) and Dr. Praveen Vemula (Institute for Stem Cell Biology and Regenerative Medicine, Bangalore, India) for helping us with TEM imaging. This work was partially supported by NTU-Northwestern Institute for Nanomedicine (To X.C.J.), the Tier-1 grant funded by the Ministry of Education in Singapore (RG41/14: 
M4011285 to M.P.), the Tier-2 grant funded by the Ministry of Education in Singapore (ARC2/15: M4020238 to M.P.). Authors have no relevant financial interests in the manuscript and no other potential conflicts of interest to disclose.

\section{References}

1. L. V. Wang and $\mathrm{S}$. Hu, "Photoacoustic tomography: in vivo imaging from organelles to organs," Science 335(6075), 1458-1462 (2012).

2. P. Beard, "Biomedical photoacoustic imaging," Interface Focus 1(4), 602-631 (2011).

3. L. V. Wang and J. Yao, "A practical guide to photoacoustic tomography in the life sciences," Nat. Methods 13(8), 627-638 (2016).

4. M. Schwarz et al., "Three-dimensional multispectral optoacoustic mesoscopy reveals melanin and blood oxygenation in human skin in vivo," J. Biophotonics 9(1-2), 55-60 (2016).

5. S. Huang et al., "A dual-functional benzobisthiadiazole derivative as an effective theranostic agent for near-infrared photoacoustic imaging and photothermal therapy," J. Mater. Chem. B 4(9), 1696-1703 (2016).

6. Y. Lyu et al., "Intraparticle molecular orbital engineering of semiconducting polymer nanoparticles as amplified theranostics for in vivo photoacoustic imaging and photothermal therapy," ACS Nano 10(4), 4472-4481 (2016).

7. K. Pu et al., "Semiconducting polymer nanoparticles as photoacoustic molecular imaging probes in living mice," Nat. Nanotechnol. 9(3), 233239 (2014).

8. D. Pan et al., "Recent advances in colloidal gold nanobeacons for molecular photoacoustic imaging," Contrast Media Mol. Imaging 6(5), 378-388 (2011).

9. C. Kim, C. Favazza, and L. V. Wang, "In vivo photoacoustic tomography of chemicals: high-resolution functional and molecular optical imaging at new depths," Chem. Rev. 110(5), 2756-2782 (2010).

10. M. Pramanik et al., "Single-walled carbon nanotubes as a multimodalthermoacoustic and photoacoustic-contrast agent," J. Biomed. Opt. 14(3), 034018 (2009).

11. C. Wiraja et al., "Near-infrared light-sensitive liposomes for enhanced plasmid DNA transfection," Bioeng. Transl. Med. (2016).

12. Y. Shi et al., "Targeted Aucore-Agshell nanorods as a dual-functional contrast agent for photoacoustic imaging and photothermal therapy," Biomed. Opt. Express 7(5), 1830 (2016).

13. J. R. Rajian et al., "Drug delivery monitoring by photoacoustic tomography with an ICG encapsulated double emulsion," Opt. Express 19(15), 14335-14347 (2011).

14. D. Pan et al., "Molecular photoacoustic imaging of angiogenesis with integrin-targeted gold nanobeacons," FASEB J. 25(3), 875-882 (2011).

15. J. Xia, C. Kim, and J. F. Lovell, "Opportunities for photoacousticguided drug delivery," Curr. Drug Targets 16(6), 571-581 (2015).

16. A. Gabizon and D. Papahadjopoulos, "Liposome formulations with prolonged circulation time in blood and enhanced uptake by tumors," Proc. Natl. Acad. Sci. U. S. A. 85(18), 6949-6953 (1988).

17. T. Sakakibara et al., "Doxorubicin encapsulated in sterically stabilized liposomes is superior to free drug or drug-containing conventional liposomes at suppressing growth and metastases of human lung tumor xenografts," Cancer Res. 56(16), 3743-3746 (1996).

18. T. Boulikas, "Clinical overview on lipoplatin ${ }^{\text {TM }}$ : a successful liposomal formulation of cisplatin," Expert Opin. Invest. Drugs 18(8), 1197-1218 (2009).

19. R. J. S. De Marie and I. Bakker-Woudenberg, "Clinical use of liposomal and lipid-complexed amphotericin B," J. Antimicrob. Chemother. 33(5), 907-916 (1994).

20. D. Needham et al., "Materials characterization of the low temperature sensitive liposome (LTSL): effects of the lipid composition (lysolipid and DSPE-PEG2000) on the thermal transition and release of doxorubicin," Faraday Discuss. 161, 515-534 (2013).

21. S. A. Abraham et al., "The liposomal formulation of doxorubicin," Methods Enzymol. 391, 71-97 (2005).

22. G. Wu et al., "Remotely triggered liposome release by near-infrared light absorption via hollow gold nanoshells," J. Am. Chem. Soc. 130(26), 8175-8177 (2008).

23. R. H. Bisby, C. Mead, and C. G. Morgan, "Wavelength-programmed solute release from photosensitive liposomes," Biochem. Biophys. Res. Commun. 276(1), 169-173 (2000).

24. M. Mathiyazhakan et al., "Non-invasive controlled release from gold nanoparticle integrated photo-responsive liposomes through pulse laser induced microbubble cavitation," Colloids Surf. B 126, 569-574 (2015).

25. S. J. Leung et al., "Wavelength-selective light-induced release from plasmon resonant liposomes," Adv. Funct. Mater. 21(6), 1113-1121 (2011).

26. A. K. Rengan et al., "Multifunctional gold coated thermo-sensitive liposomes for multimodal imaging and photo-thermal therapy of breast cancer cells," Nanoscale 6(2), 916-923 (2014).

27. A. K. Rengan et al., "In vivo analysis of biodegradable liposome gold nanoparticles as efficient agents for photothermal therapy of cancer," Nano Lett. 15(2), 842-848 (2015).

28. J. K. Mills and D. Needham, "Lysolipid incorporation in dipalmitoylphosphatidylcholine bilayer membranes enhances the ion permeability and drug release rates at the membrane phase transition," Biochim. Biophys. Acta 1716(2), 77-96 (2005).

29. P. K. Upputuri and M. Pramanik, "Performance characterization of lowcost, high-speed, portable pulsed laser diode photoacoustic tomography (PLD-PAT) system," Biomed. Opt. Express 6(10), 4118-4129 (2015).

30. K. Sivasubramanian and M. Pramanik, "High frame rate photoacoustic imaging at 7000 frames per second using clinical ultrasound system," Biomed. Opt. Express 7(2), 312-323 (2016).

31. R. Chen et al., "Fabrication of gold nanoparticles with different morphologies in HEPES buffer," Rare Met. 29(2), 180-186 (2010).

32. W. T. Ferrar et al., "Metalization of lipid vesicles via electroless plating," J. Am. Chem. Soc. 110(3), 288-289 (1988).

33. M. R. Hamblin and Y. Y. Huang, Eds., Handbook of Photomedicine, Taylor \& Francis, Boca Raton, Florida (2013).

34. A. Sazgarnia et al., "Therapeutic effects of acoustic cavitation in the presence of gold nanoparticles on a colon tumor model," J. Ultrasound Med. 32(2), 475-483 (2013).

Biographies for the authors are not available. 